\section{Bendamustin + Rituximab in der Therapie indolenter NHL und Mantelzell-Lymphome - hoch effektiv und gut verträglich}

Bendamustin, eine seit langem bewährte Substanz, hat sich in der Behandlung der indolenten Lymphome als Monosubstanz, aber auch in Kombination mit dem CD20Antikörper Rituximab als hoch effektiv und sehr gut verträglich erwiesen. Neueste Studiendaten, die auf dem diesjährigen ASCO-Kongress präsentiert wurden und nun auch im J Clin Oncol 2005:23(15) erschienen sind, festigen den Stellenwert von Bendamustin im Rezidiv und bieten interessante Perspektiven für neu diagnostizierte Patienten mit indolenten Non-HodgkinLymphomen (NHL).

Auf der Basis von in-vitro-Daten, die eine synergistische Wirkung von Bendamustin und Rituximab gezeigt hatten, untersuchten Rummel et al. [J Clin Oncol 2005] diese BR-Kombination im Rahmen einer Phase-II-Studie bei rezidivierten und refraktären indolenten NHL und MantelzellLymphomen. Ein Update der Daten wurde auf dem ASCO-Kongress präsentiert: 63 rezidivierte NHL-Patienten erhielten: Bendamustin $\left(90 \mathrm{mg} / \mathrm{m}^{2}\right.$ Tag $2+3 \mathrm{q} 4 \mathrm{w}, 4 \mathrm{Zy}$ klen) in Kombination mit Rituximab (375 $\mathrm{mg} / \mathrm{m}^{2}$ Tag $\left.1 \mathrm{q} 4 \mathrm{w}\right)$. Ein Drittel der Patienten hatte auf die Vortherapie nicht angesprochen (refraktäre Patienten).

Die Ansprechrate auf das BR-Regime war mit $90 \%$ sehr hoch. Von den 16 Patienten mit Mantelzell-Lymphom sprachen 75\% auf das BR-Regime an. Die Remissionsdauer liegt bei 30 Monaten, das 4-JahresÜberleben bei $55 \%$. Das BR-Regime wurde insgesamt sehr gut vertragen.

Basierend auf diesen Erfolg versprechen- den Daten vergleicht die StiL (Studiengruppe indolente Lymphome, Frankfurt) in zwei Phase-III-Studien das BR-Regime gegen Standardregime: In der Primärtherapie wird BR gegen CHOP-R randomisiert, im Rezidiv BR gegen FR (Fludarabin + Rituximab).

\section{BMR-Kombination - effektiv auch nach} einer Rituximab-haltigen Vortherapie

Die Kombination von Bendamustin und Rituximab, erweitert um die Substanz Mitoxantron zum BMR-Schema, wurde von Weide et al. [2004] untersucht. Aufgrund der viel versprechenden Pilotdaten dieser BMR-Kombination bei rezidivierten indolenten NHL und CLL initiierte die Deutsche Studiengruppe für indolente Lymphome (GLSG) eine multizentrische BMRRezidivstudie, deren update nun auf dem ASCO von Weide et al. präsentiert wurde: Bendamustin $\left(90 \mathrm{mg} / \mathrm{m}^{2} \mathrm{Tag} 1+2\right)$ wurde mit Mitoxantron $\left(10 \mathrm{mg} / \mathrm{m}^{2}\right.$ Tag 1$)$ und Rituximab (375 mg/m² Tag 8) q4w für bis zu 4 Zyklen gegeben. $40 \%$ der Patienten waren bereits mit Rituximab-haltigen Schemata vorbehandelt. Die Therapie konnte ambulant durchgeführt werden und wurde gut vertragen: Die nicht-hämatologischen Toxizitäten beschränkten sich meist auf Grad 1/2; als Grad-3/4-Toxizitäten traten reversible hämatologische Nebenwirkungen bei $80 \%$ der Patienten auf.

Die BMR-Kombination erwies sich als außerordentlich effektiv: Von den 47 auswertbaren rezidivierten NHL-Patienten sprachen $93 \%$ auf BMR an. Bei den $\mathrm{Pa}-$ tienten mit Mantelzell-Lymphom konnte eine Remission bei $88 \%$ der Patienten erreicht werden. Auch von den 19 Patienten, die bereits mit Rituximab vorbehandelten waren, sprachen $89 \%$ auf BMR an.
Bendamustin bei Rituximab-refraktären NHL-Patienten

Neben den Kombinations-Schemata zeigt Bendamustin auch als Monosubstanz viel versprechende Aktivität bei NHL-Patienten, die auf eine Rituximab-haltige Therapie nicht oder nur kurz (weniger als 6 Monate) angesprochen hatten: Im Rahmen einer zulassungsrelevanten Phase-II-Studie in den USA (Cohen et al.) wurde Bendamustin in einer Dosierung von $120 \mathrm{mg} / \mathrm{m}^{2}$ an Tag $1+2$, q3w, eingesetzt. Von den 32 auswertbaren Patienten sprachen insgesamt $75 \%$ auf Bendamustin an, davon 25\% mit einer CR. Die Haupttoxizitäten waren Grad-3/4-Neutropenie bei 39\% der Patienten und Grad-3/4-Thrombopenie bei 19\% der Patienten.

\section{Fazit}

Bendamustin in Kombination mit dem CD20-Antikörper Rituximab (BR-Regime) ist eine sehr effektive Therapie mit hohen Ansprechraten und langer Remissionsdauer für indolente NHL, aber auch Mantelzell-Lymphome. Die Kombination wird gut vertragen, die Toxizität beschränkt sich vorwiegend auf hämatologische Nebenwirkungen. Auch bei Rituximab-vortherapierten Patienten erweist sich die BMR-Kombination mit einer Ansprechrate von etwa $90 \%$ als sehr effektiv. Für NHL-Patienten, die gar nicht oder nur kurz auf Rituximab angesprochen hatten, bietet sich Bendamustin zudem als Monotherapie an.

Weitere Informationen bei

Dr. Almut-J. Wegner

ribosepharm $\mathrm{GmbH}$

Tel. +49 8945450 201, Fax -266

E-mail almut.wegner@ribosepharm.de

\title{
PharmaTicker+++ PharmaTicker+++ PharmaTicker+++ PharmaTicker+++
}

Gilead Sciences GmbH. Die jetzt vorliegenden 144-Wochen-Daten $(n=309)$ der auf 5 Jahre ausgelegten Nachbeobachtung der Hepsera ${ }^{\circledR}$ Zulassungsstudie mit HbeAg-positiven Patienten zeigen, dass durch die Behandlung mit Adefovirdipivoxil eine signifikante Verringerung der HBV-DANN-Spiegel erreicht wurde.

Weitere Informationen bei

Gilead Sciences $\mathrm{GmbH}$

Dr. Diane Langenbacher-Nießing, Conrad Fischer

Tel.: +49 89 899890-54, Fax -90

E-Mail dlangenbacher@gilead.com,

cfischer@gilead.com
ALTANA Pharma Deutschland GmbH/Abbott GmbH. Da Compliance für den Therapieerfolg entscheidend ist, muss die Behandlung so einfach wie möglich gestaltet werden. Bei einer komplexen Therapie wie der Eradikation von Helicobacter pylori bietet sich daher die intelligent aufgebaute und patientenfreundliche Kombinationspackung $\left(\mathrm{ZacPac}^{\circledR}\right)$ als kostengünstiges 7-tägiges Behandlungsregime an. Weitere Informationen bei ALTANA Pharma Deutschland GmbH

Moltkestr. 4, 78467 Konstanz Abbott GmbH \& Co. KG

Postfach 2106 60, 67006 Ludwigshafen
Merck Pharma GmbH. Die neuen vorläufigen Ergebnisse einer internationalen Phase-II-Studie (ACROBAT) zeigen eine objektive Ansprechrate von $81 \%$ in der First-line-Therapie des metastasierten kolorektalen Karzinoms mit der Kombinationstherapie aus Erbitux und FOLFOX-4. Dadurch wurde ein progressionsfreies Überleben über 12 Monate hinaus erreicht. Weitere Informationen bei Intramedic, Dr. Cornelia Bartels

Tel. +49 6102 7993-338, Fax -301

E-mail cornelia_bartels@de.yr.com

\begin{tabular}{ll}
\hline KARGER & @ 2005 S. Karger GmbH, Freiburg \\
& \\
$\begin{array}{l}\text { Fax }+497614520714 \\
\begin{array}{l}\text { E-mail Information@Karger.de } \\
\text { www.karger.com }\end{array}\end{array}$ & $\begin{array}{l}\text { Accessible online at: } \\
\text { www.karger.com/onk }\end{array}$ \\
\end{tabular}




\section{DRTHD BIDTECH}

DIVISION OF JANSSEN-CILAG GmbH

\section{Multiples Myelom: Velcade ${ }^{\circledR}$ erhält erweiterte Zulassung}

Ortho Biotech, Division of Janssen-Cilag, hat im April 2005 von der Europäischen Kommission die erweiterte Zulassung für Velcade ${ }^{\circledR}$ (Bortezomib) zur Behandlung des multiplen Myeloms in Europa erhalten. Bisher beschränkte sich die Therapie auf Patienten mit progressivem multiplem Myelom nach mindestens zwei vorangegangenen Behandlungen.

Mit der erweiterten Zulassung kann Velca$\mathrm{de}^{\circledR}$ jetzt als Rezidivtherapie bei Patienten mit nur einer Vorbehandlung eingesetzt werden. Die Patienten sollten sich bereits einer Knochenmarktransplantation unterzogen haben oder sich für diese nicht eignen, um Velcade ${ }^{\circledR}$ als Monotherapie zu erhalten.

Basis der Zulassungserweiterung sind die positiven Daten der Phase-III-Studie APEX [Assessment of Proteasome-Inhibition for Extending Remissions; Richardson P. et al.: Blood 104 (11)], die im März 2005 $\mathrm{zu}$ einer erweiterten Zulassung in den USA durch die FDA geführt haben. In dieser bisher größten randomisierten Studie beim rezidivierten multiplen Myelom wurde Velcade ${ }^{\circledR}$ bei 669 Patienten mit 1-3 Vortherapien gegenüber hochdosiertem Dexamethason verglichen. Dabei zeigte sich ein signifikanter Überlebensvorteil für die mit dem Proteasom-Inhibitor behandelten Patienten. So hat die Analyse unter Bortezomib eine Verlängerung der Zeit bis zum Fortschreiten der Erkrankung um $78 \%$ ergeben. Auch die Remissionsrate (CR + PR) war mit 38\% bei den mit Velca$\mathrm{de}^{\circledR}$ behandelten Patienten signifikant höher als beim Dexamethason-Arm mit $18 \%$. Prof. Jesus San Miguel, Salamanca/ Spanien: «Nach einem Jahr unter Bortezomib lebten noch $80 \%$ der Patienten gegenüber $66 \%$ der Patienten des Dexamethason-Arms. Dies entspricht einem signifikant reduzierten Mortalitätsrisiko von 41\%.» Hervorzuheben ist zudem, dass der Überlebensvorteil bei den Rezidivpatienten mit nur einer Vortherapie besser ausfiel.

Weitere Informationen bei

Kerstin Aschoff

Janssen-Cilag GmbH

Tel. +49 2137 955-412

E-mail kaschoff@jacde.jnj.com

\section{Shire Deutschland mit Spezial- Präparaten auf Expansionskurs}

Shire, der Global Player für innovative, wissenschaftlich fundierte Spezialpräparate, baut seine Position auf dem deutschen Markt deutlich aus. «Mit zwei ProduktLaunches in den Indikationen Dermatologie und Onkologie will Shire Deutschland seinen Umsatz in diesem Jahr verdoppeln», gibt Leonhard Terp, Geschäftsführer Shire Deutschland, bekannt.

Shire engagiert sich seit der Akquisition von Fuisz-Pharma im Jahr 1999 auf dem deutschen Pharmamarkt. Zum Portfolio gehörten zunächst fünf Arzneimittel aus dem Bereich Herz-Kreislauf-Erkrankungen. Mit Eintritt von Leonhard Terp im Jahre 2002 konzentrierte sich Shire
Deutschland auf wissenschaftlich fundierte Spezialpräparate mit eindeutigem Profil. Im Januar diesen Jahres führte Shire Deutschland das inzwischen EU-weit zugelassene Präparat Xagrid ${ }^{\circledR}$ (Wirkstoff: Anagrelid) gegen essentielle Thrombozythämie in den deutschen Markt ein. Xagrid ${ }^{\circledR}$ wirkt plättchenselektiv und beeinflusst die Reifung, Größe und Ploidie der Knochenmarksriesenzellen, sodass weniger Plättchen gebildet werden. In puncto Wirksamkeit ist es mit dem Goldstandard Hydroxyurea vergleichbar. Xagrid ${ }^{\circledR}$ hat seine Langzeitsicherheit an 3660 Patienten unter Beweis gestellt. Unter Xagrid ${ }^{\circledR}$ treten hier keine erhöhten Transformationsraten zur akuten Leukämie auf.

Speziell für die Behandlung aktinischer Keratosen, die die Deutsche Gesellschaft für Dermatologie inzwischen nicht mehr als Präkanzerosen, sondern als Karzinoma in situ klassifiziert, entwickelte Shire das Präparat Solaraze ${ }^{\circledR}$-Gel: In Hyaluronsäure gelöstes Diclofenac beseitigt aktinische Keratosen effektiv und patientenfreundlicher als herkömmliche Methoden wie Vereisung oder Curretage. In der Klinik zeigte sich eine hohe therapeutische Wirksamkeit von Solaraze ${ }^{\circledR}$ bei zweimal täglicher Anwendung über 60-90 Tage. Die Behandlung ist gut verträglich, es kommt unter Solaraze $^{\circledR}$ nicht $\mathrm{zu}$ Narbenbildung oder Schmerzen.

Weitere Informationen bei

Shire Deutschland GmbH \&Co.KG

Siegburger Str. 126

50679 Köln

Tel. +49221880 47-30

\section{PharmaTicker+++ PharmaTicker+++ PharmaTicker+++ PharmaTicker+++}

Hoffmann-La Roche AG. Bevacizumab (Avastin) hat sich nun neben Darmkrebs auch bei Brust- und Lungenkrebs als hochwirksam erwiesen - das zeigen Studiendaten, die jetzt auf der Jahrestagung der American Society of Clinical Oncology (ASCO) vorgestellt wurden. Damit stellt der Angiogenese-Hemmer bei den drei weltweit häufigsten Krebsarten eine bedeutende Verbesserung der Therapie dar.

Weitere Informationen bei

Hoffmann-La Roche AG, Dr. med. H.-U. Jelitto

Tel. +49 7624 14-2400, Fax -3366

www.roche.de
Adept Scientific GmbH. Die Adept Scientific GmbH hat die Software StudyBuilder in ihr wissenschaftlich-technisches Produktportfolio aufgenommen. StudyBuilder ist mit über 50.000 Nutzern, über 3 Millionen Studiensubjekten in über 40 Ländern die weltweit führende Software zur Planung, Verwaltung und Durchführung von klinischen oder medizinischen Studien.

Weitere Informationen bei

Adept Science GmbH, Falko Schumacher

Tel. +49 69 970841-0, Fax -41

E-mail pr@adeptscience.de
Sirtex Medical Europe GmbH. Das Institut für Entgeltsysteme im Krankenhaus (InEK) stimmt der individuellen Verhandlung zehn deutscher Krankenhäuser mit den gesetzlichen Krankenkassen zur Erstattung der selektiven internen Radiotherapie (SIRT) von Lebertumoren mit Yttrium-90 markierten Mikrosphären zu. Weitere Informationen bei ipse Communication $\mathrm{GmbH}$, Katrin Thormeier Tel. +49 30 288846-14 E-mail k.thormeier@ipse.de

\begin{tabular}{|c|c|}
\hline KARGER & (ㄱ) 2005 S. Karger GmbH, Freiburg \\
\hline $\begin{array}{l}\text { Fax + 497614520714 } \\
\text { E-mail Information@Karger.de } \\
\text { www.karger.com }\end{array}$ & $\begin{array}{l}\text { Accessible online at: } \\
\text { www.karger.com/onk }\end{array}$ \\
\hline
\end{tabular}




\section{GlaxoSmithKline}

\section{Therapiemanagement des Ovarial- karzinoms mit Hycamtin ${ }^{\circledR}$}

Der Topoisomerasehemmer Topotecan $\left(\right.$ Hycamtin $\left.{ }^{\circledR}\right)$ ist eine wirksame Therapieoption für Patientinnen mit fortgeschrittenem epithelialem Ovarialkarzinom, die nach Primärbehandlung mit Platin/Paclitaxel rezidivieren. Klassischerweise wird Hycamtin ${ }^{\circledR}$ an 5 aufeinander folgenden Tagen als Bolus-Injektion appliziert. Ergebnisse klinischer Studien deuten jedoch daraufhin, dass auch mit der wöchentlichen Applikation von Topotecan eine wirksame Therapie möglich ist, die von den Patientinnen gut toleriert wird.

Rezidivierte Patientinnen mit fortgeschrittenem Ovarialkarzinom haben eine sehr ungünstige Prognose. Im Mittelpunkt der Therapie steht die Verlängerung der Überlebenszeit bei guter Lebensqualität. Haben die Patientinnen auf die Primärbehandlung mit Platin/Paclitaxel nicht adäquat angesprochen, ist die Monotherapie mit Hycamtin ${ }^{\circledR}$ eine wirksame und sichere Therapieoption. Etwa ein Drittel der Patientinnen erreicht unter Hycamtin ${ }^{\circledR}$ eine objektive Tumorremission (Tumorrückbildung $>50 \%$ ). Bei bis zu $50 \%$ der Patientinnen kommt es vorübergehend zum Wachstumsstillstand des Tumors. In einer randomisierten Phase-III-Vergleichsstudie mit Hycamtin ${ }^{\circledR}$ versus Treosulfan schlug sich dies auch in einem statistisch signifikanten Überlebensvorteil zugunsten der mit Topotecan (Hycamtin ${ }^{\circledR}$ ) behandelten Patientinnen nieder. Letztere überlebten im Median 2 Monate länger (13 vs. 11 Monate; $\mathrm{p}=$ 0,0235; AGO-Ovar 2.3-Studie).
Wöchentliche Gabe von Topotecan als weitere Therapieoption

Topotecan wird klassischerweise in einer Dosierung von $1,25-1,5 \mathrm{mg} / \mathrm{m}^{2}$ an 5 aufeinander folgenden Tagen (Tag 1-5), alle 21 Tage, appliziert. Hauptnebenwirkung ist eine Myelosuppression, die von den $\mathrm{Pa}$ tientinnen subjektiv nicht bzw. kaum wahrgenommen wird. Klinisch hat die Myelosuppression in der Regel keine Relevanz: febrile Neutropenien oder Infektionen sind selten.

Phase-I/II-Studien haben nun gezeigt, dass die wöchentliche Gabe von Topotecan eine wirksame Therapiealternative zur täglichen Infusion über 5 Tage sein könnte. Die wöchentliche Gabe zeigt eine vergleichbare Wirksamkeit, ihr Vorteil ist die einfachere Logistik für die Therapie.

\section{Gute Wirksamkeit und Verträglichkeit} unter der wöchentlichen Gabe

In einer multizentrischen Phase-II-Studie einer US-amerikanischen Studiengruppe (Morris et al., Proc ASCO 2002; 21:173b; Update ASCO 2003) erreichten $28 \%$ der Patientinnen mit fortgeschrittenem epithelialem Ovarialkarzinom unter der wöchentlichen Gabe von Topotecan eine objektive Remission (7/26). Alle Patientinnen waren mit einer Platin/Taxan-haltigen Chemotherapie vorbehandelt. Die Patientinnen mit objektiver Remission blieben im Median 28,3 Wochen ohne erneutes Tumorwachstum. Mit im Median 7 Wochen bis zum maximalen Therapieerfolg sprachen sie sehr schnell auf die Behandlung an. 6 weitere Patientinnen erzielten eine Stabilisierung ihrer Erkrankung; sie blieben bis zu 32 Wochen ohne erneute Tumorprogression (5-32 Wochen).

Die Patientinnen hatten pro Woche 4,0 $\mathrm{mg} / \mathrm{m}^{2}$ Topotecan erhalten. Therapieverzö- gerungen waren in lediglich $13 \%$ aller Behandlungszyklen notwendig und damit sehr selten, davon $9 \%$ wegen hämatologischer Ereignisse. Es traten keine Grad-3/4Thrombozytopenien und keine Grad-4Anämien auf. Lediglich eine Patientin entwickelte eine Grad-4-Neutropenie. Die Inzidenz schwerer nicht-hämatologischer Nebenwirkungen war ebenfalls extrem niedrig: Zwei Patientinnen entwickelten eine Grad-3-Fatigue und eine Patientin hatte gastrointestinale Probleme (Grad 3).

Die Ergebnisse sind ein wichtiger Hinweis darauf, dass die wöchentliche Gabe von Topotecan eine Therapiealternative für $\mathrm{Pa}$ tientinnen mit rezidiviertem epithelialem Ovarialkarzinom sein können.

\section{Randomisierte Phase-II-Studie der} NOGGO

Aufgrund der viel versprechenden Ergebnisse mit der wöchentlichen Gabe von Topotecan vergleicht die NOGGO (NordOstdeutsche Gesellschaft für Gynäkologische Onkologie) derzeit im Rahmen einer randomisierten Phase-II-Studie bei Platinresistenten Patientinnen mit fortgeschrittenem Ovarialkarzinom die wöchentliche Infusion von 4,0 mg/m² Hycamtin ${ }^{\circledR}$ (Tag 1, 8, 15), alle 28 Tage, mit der 5-tägigen Applikation von $1,25 \mathrm{mg} / \mathrm{m}^{2}$ Hycamtin $^{\circledR}$, alle 21 Tage. Platin-resistente Patientinnen haben eine besonders ungünstige Prognose. Hier stehen die Verträglichkeit der Behandlung und die davon abhängende Lebensqualität im Vordergrund.

Weitere Informationen bei

GlaxoSmithKline

Dr. Charlotte Stein

Theresienhöhe 11, 80339 München

Tel.: +4989360448479

Email charlotte.stein@gsk.com

\section{PharmaTicker+++ PharmaTicker+++ PharmaTicker+++ PharmaTicker+++}

MedacSchering Onkologie GmbH. Der frühzeitige Einsatz von Zevalin ${ }^{\circledR}\left({ }^{90}\right.$ Yttrium-Ibritumomab-Tiuxetan) bei rezidivierenden follikulären Lymphomen als Second-line-Therapie gilt nach den Empfehlungen der Amerikanischen Gesellschaft für Hämatologie (ASH) momentan als die beste Therapieoption. Neue Daten vom Amerikanischen Krebskongress (ASCO) bestätigen dieses Konzept.

Weitere Informationen bei

Melitta A. Zakel

Kommunikation und Beratung im Gesundheitswesen Tel. +49 89 159-6752, Fax -70492

E-mail zakel.pr@t-online.de
MSD Sharp \& Dohme GmbH. Bei Patienten, die mit Ciclosporin A behandelt wurden, bereitete eine Salvage-Therapie mit Caspofungin $\left(\right.$ Cancidas $\left.^{\circledR}\right)$ keine Probleme und war meist erfolgreich. Dies zeigte $u$. a. eine kürzlich in «Bone Marrow Transplantation» publizierte retrospektive Analyse von 23 Patienten eines Kollektivs von insgesamt 31 mit Caspofungin behandelten Patienten mit invasiver Mykose oder Fieber unklarer Genese nach allogener Stammzelltransplantation, bei denen die Primärtherapie mit Amphotericin B oder Azolen versagt hatte. MSD Sharp \& Dohme GmbH, Anette Menzel Tel. +49 89 45611-353, Fax -329

E-mail anette_menzel@msd.de
Genzyme GmbH. Im Februar hat die Europäische Zulassungsbehörde die Zulassung des rekombinanten humanen Thyrotropin alfa (rhTSH, Thyrogen ${ }^{\circledR}$ ) um eine therapeutische Indikation erweitert. Danach kann rhTSH zusammen mit Radiojod (I-131) jetzt auch bei der Ablation nach Thyreoidektomie zur Erhöhung der TSHSpiegel ohne Schilddrüsen-Unterfunktion eingesetzt werden.

Weitere Informationen bei

Genzyme GmbH, Sabine Grätz

Tel. +49 6102 3674-592

E-mail sabine.graetz@genzyme.com

\begin{tabular}{ll}
\hline KARGER & @ 2005 S. Karger GmbH, Freiburg \\
& \\
$\begin{array}{l}\text { Fax }+497614520714 \\
\begin{array}{l}\text { E-mail Information@Karger.de } \\
\text { www.karger.com }\end{array}\end{array}$ & $\begin{array}{l}\text { Accessible online at: } \\
\text { www.karger.com/onk }\end{array}$ \\
\end{tabular}




\section{(9) \\ Pierre Fabre Pharma ONKOLOGIE}

\section{NSCLC: Längeres Überleben durch adjuvante Therapie mit Navelbine + Cisplatin}

Bei der Behandlung von Patienten mit einem nicht-kleinzelligen Bronchialkarzinom (NSCLC) in frühen Stadien ist meist eine komplette chirurgische Resektion Therapie der Wahl. Der Stellenwert einer adjuvanten Therapie für diese Patienten war bis vor kurzem durch eine heterogene Datenlage geprägt und stellte daher noch keine gesicherte Indikation.

Patienten profitieren in Stadien II und IIIA Auf dem diesjährigen ASCO konnte die sogenannte ANITA-Studie (Adjuvant Navelbine International Trialist Association), eine prospektiv randomisierte Phase-IIIStudie mit über 800 Patienten aufzeigen, dass diese nach vollständiger Resektion (R0) und einer zusätzlichen adjuvanten Therapie eindeutig profitieren (J.Y. Douillard et al., ASCO 2005, Abstract-Nr.: 7013).

Einschlusskriterien in die Studie waren gegeben durch: histologisch gesichertes NSCLC, TNM-Stadien IB, II, IIIA, vollständig reserziert (R0) innerhalb 42 Tage und ein guter Allgemeinzustand der $\mathrm{Pa}$ tienten.

370 Patienten erhielten nach Resektion eine adjuvante Chemotherapie, bestehend aus Cisplatin $100 \mathrm{mg} / \mathrm{m}^{2}$ i.v. (T1, T29, T57, T85) und Navelbine $30 \mathrm{mg} / \mathrm{m}^{2}$ i.v. (wöchentlich, 16 Gaben in 20 Wochen), 470 Patienten wurden nach Operation observiert. Beide Arme der Studie waren hinsichtlich des Ausmaßes der Resektion, Histologie und TNM-Staging gut ausbalanciert.

Nach einer Follow-up-Zeit von über 70 Monaten zeigten sich vor allem bei $\mathrm{Pa}$ tienten in den Stadien II und IIIA signifikant verbesserte 5-Jahres-Überlebensraten, während Patienten im Stadium IB keine signifikanten Überlebensunterschiede in beiden Armen verzeichneten. Die 5-Jahres-Überlebensrate von Patienten im Stadium II beträgt im Adjuvanzarm 52\% und im Observationsarm 39\% und im Stadium IIIA 42 bzw. 26\%. Das Gesamtüberleben nach 7 Jahren beträgt für Patienten unter Therapie mit Cisplatin/Navelbine 45\% und im Observationsarm 37\%.

\section{Datenlage verdichtet}

Die Heterogenität der bis zu diesem Zeitpunkt vorliegenden Datenlage kann darauf zurückgeführt werden, dass in älteren Studien Patienten adjuvant meist mit Zytostatika der ersten Generation behandelt wurden, während in kürzlich publizierten Studien platinhaltige Kombinationen mit $\mathrm{Pa}$ clitaxel (Strauss et al., ASCO 2004) oder Navelbine (Le Chevalier et al., ASCO 2003; Winton et al., ASCO 2004; Douillard et al., ASCO 2005) eingesetzt wurden. Letztere Studien zeichnen sich in einem signifikant verbesserten medianen Überleben als auch 5-Jahres-Überleben aus.
Der Evidenz-basierte Überlebensvorteil durch eine adjuvante Chemotherapie mit Navelbine + Cisplatin konnte nach der Veröffentlichung der ANITA-Studie weiter untermauert werden.

\section{Vinorelbin oral}

Da die Therapie-Compliance in ANITA nicht optimal war, wurde überlegt, wie sich hier eine Verbesserung erreichen lässt. Vinorelbin ist seit September 2003 auch in oraler Form zugelassen, und die pharmakokinetische Bioäquivalenz mit dem intravenös verabreichten Vinorelbin ist erwiesen. Deshalb dürfte es für die Patienten angenehmer sein, Vinorelbin an den Cisplatin-freien Tagen nicht intravenös zu erhalten, sondern einfach zu schlucken, was zu Hause möglicht ist, aber auch unter Aufsicht in der Praxis erfolgen kann, und demnoch allen Beteiligten viel Zeit und Aufwand spart und dadurch die Compliance steigern dürfte.

Weitere Informationen bei PIERRE FABRE PHARMA GMBH Jechtingerstr. 13

79111 Freiburg

Tel. +49 761 45261-0, Fax -44

\section{PharmaTicker+++ PharmaTicker+++ PharmaTicker+++ PharmaTicker+++}

Bayer HealthCare AG. Sorafenib (früher BAY 43-9006) von der Bayer HealthCare AG und Onyx Pharmaceuticals Inc. hat für die Behandlung des fortgeschrittenen Nierenkarzinoms im März 2004 den Fast Track Status erhalten und ist nun in den USA in das Pilot-1-Programm der Food and Drug Administration (FDA) aufgenommen worden.

Weitere Informationen be

Bayer HealthCare AG, Dr. Michael S. Diehl

Tel. +49 2143058532

E-mail michael.diehl@bayerhealthcare.com
Micromet AG. Die Micromet AG hat eine klinische Studie der Phase I begonnen, um ihren humanen Antikörper MT201 (Adecatumumab) in Kombination mit Taxotere ${ }^{\circledR}$ (Docetaxel) zur Behandlung von Brustkrebs zu testen. Zielgruppe sind Patientinnen, die bereits einen Rückfall ihrer Erkrankung erlitten haben.

Weitere Informatione bei Micromet AG, Evelyn Wolf Tel. +49 $89895277-220$ E-mail evelyn.wolf@micromet.de
Janssen-Cilag GmbH. Eine Anwendungsbeobachtung zur Therapie mit Fentanyl-TTS (Durogesic $^{\circledR}$ SMAT) bei chronischen Schmerzen zeigt, dass die Schmerzreduktion mit transdermalem Fentanyl die individuell wahrgenommene Lebensqualität im Vergleich zur vorherigen Therapie verbessert.

Weitere Informationen bei

Publicis Vital PR, Astrid Herzog

Tel. +49 221 912719-48, Fax -7

E-mail astrid.herzog@publicis-vital-pr.de

\begin{tabular}{|c|c|}
\hline KARGER & (C) 2005 S. Karger GmbH, Freiburg \\
\hline $\begin{array}{l}\text { Fax + } 497614520714 \\
\text { E-mail Information@Karger.de } \\
\text { www.karger.com }\end{array}$ & $\begin{array}{l}\text { Accessible online at: } \\
\text { www.karger.com/onk }\end{array}$ \\
\hline
\end{tabular}




\section{Pfizers SU11248 kann das Überleben bei Glivec ${ }^{\circledR}$-resistenten GIST-Patienten verlängern}

Mehr als eine Verdopplung der Überlebensdauer von Patienten mit Glivec ${ }^{\circledR}$-resistenten, gastrointestinalen Stromatumoren (GIST) konnte in einer Phase-III-Studie mit dem Wirkstoff SU11248 von Pfizer verzeichnet werden. Das Wachstum und die Ausbreitung des Tumors wurden signifikant verringert. Auch bei anderen Tumortypen wurden ermutigende Phase-II-Ergebnisse erzielt, u. a. beim metastasierten Nierenzellkarzinom, metastasierten Brustkrebs und bei neuroendokrinen Tumoren. Dies ergaben neue Daten, die jetzt auf dem Jahrestreffen der American Society of Clinical Oncology (ASCO) präsentiert wurden.

SU11248 ist ein hochselektiver, Multi-Target-Tyrosinkinase-Inhibitor, der Tumoren Blut und Nährstoffe, die für das Wachstum nötig sind, vorenthält und gleichzeitig Tumorzellen abtötet.

\section{Studien zum gastrointestinalen}

Stromatumor

Die Ergebnisse der GIST Phase-III-Studie bestärken die Annahme, dass die MultiTarget-Therapie das Problem der Resistenzentwicklung von nur auf einzelne Targets (Ziele) gerichteten Medikamenten überwinden kann.

- Ergebnisse einer doppelblinden PhaseIII-Studie mit über 300 GIST-Patienten, die gegen eine Standardbehandlung mit Imatinib $\left(\right.$ Glivec $\left.^{\circledR}\right)$ resistent waren oder diese nicht tolerierten, zeigen, dass SU11248 die Zeit bis zum erneuten Tumorwachstum signifikant verlängerte $(6,3 \mathrm{Mo}$ - nate mit SU11248 vs. 1,5 Monate in der Kontrollgruppe).

- Zusätzlich zeigen Langzeit-Kontrolldaten aus der Phase-I/II-GIST-Studie, dass sich das Überleben bei Patienten, deren Krebs trotz anderer Standardtherapien weiter gewachsen war, auf fast 20 Monate erhöhte. Die mediane Zeit bis zum erneuten Tumorwachstum betrug 7,8 Monate bei allen Patienten.

«Diese Ergebnisse untermauern die Auffassung, dass die Multi-Target-Therapie die Resistenz gegenüber anderen zielgerichteten Medikamenten bei Krebs überwinden kann», sagte Dr. George Demetri vom Dana-Farber Cancer Institute der Harvard University in Boston, Forschungsleiter der SU11248-Studie für GIST. «Wir glauben, dass SU11248 ein breites Aktivitätsspektrum bei vielen verschiedenen Krebsarten haben könnte, das über jenes, das wir bei GIST-Patienten beobachtet haben, hinausgeht. Wir glauben, dass SU11248 ein wichtiger Schritt vorwärts in der Krebstherapie ist.»

\section{Studien zum metastasierten}

Nierenzellkarzinom

Die Ergebnisse aus diesen Studien weisen nach, dass SU11248 eine substanzielle Anti-Tumor-Wirkung bei metastasiertem Nierenzellkarzinom als Zweitlinientherapie nach Versagen der Standardtherapie mit Zytokinen hat. Sie sind Grundlage für eine breit angelegte Phase-III-Studie zur Bestimmung der potenziellen Vorteile von SU11248 in einem früheren Erkrankungsstadium.

- Die Ergebnisse einer Studie mit $63 \mathrm{~Pa}$ tienten zeigen, dass $40 \%$ der Patienten gemäß Standardkriterien auf die Behandlung mit SU11248 ansprachen. Die Tumoren vergrößerten sich bei weiteren $28 \%$ der Patienten in mehr als 3 Monaten nicht. Insgesamt profitierten $68 \%$ der eingeschlossenen Patienten von der Behandlung mit SU11248. Die durchschnittliche Zeit bis zum erneuten Tumorwachstum betrug 8,7 Monate, das mediane Gesamtüberleben 16,4 Monate.

- Eine zweite Phase-II-Studie mit $106 \mathrm{~Pa}$ tienten weist eine objektive Ansprechrate von 39\% und einen Stillstand des Tumorwachstums bei $23 \%$ der mit SU11248 behandelten Patienten nach. Insgesamt profitierten $62 \%$ der Probanden von einer Behandlung mit SU11248.

\section{Vorläufige Daten zu neuroendokrinen} Krebsarten und Brustkrebs

Auch die vorläufigen Ergebnisse mit SU11248 bei inoperablen neuroendokrinen Tumoren und vielfach vorbehandeltem metastasiertem Brustkrebs sind viel versprechend:

- Erste Ergebnisse einer Phase-II-Studie mit SU11248 bei inoperablen neuroendokrinen Tumoren zeigen, dass bei $82 \%$ der behandelten Patienten ein Stillstand des Tumorwachstums eintrat.

- In einer Phase-II-Studie wurden Patientinnen mit metastasiertem Brustkrebs, die nach größtenteils mehr als zwei Vortherapien wieder progredient waren, untersucht. Die Monotherapie mit SU11248 führte bei $16 \%$ dieser multipel vorbehandelten Patientinnen in 1\% zum Rückgang oder Stillstand der Erkrankung.

Weitere Informationen bei

Edelman GmbH

Dr. Raphaela Farrenkopf / Martin Flörkemeier Bettinastr. 64

60325 Frankfurt am Main

Tel. +49 69 756199-75/-49, Fax -13

E-mail raphaela.farrenkopf@edelman.com/

martin.floerkemeier@edelman.com

\section{PharmaTicker+++ PharmaTicker+++ PharmaTicker+++ PharmaTicker+++}

Hoffmann-La Roche AG. Die Verabreichung von Rituximab (MabThera ${ }^{\circledR}$ ) als Erhaltungstherapie nach Beendigung einer Einstiegsbehandlung mit Rituximab plus Chemotherapie verdoppelt nahezu die Remissionsdauer von Lymphom-Patienten. Diese aktuellen Studienergebnisse wurden im Mai auf der Jahrestagung der American Society of Clinical Oncology (ASCO) vorgestellt.

Weitere Informationen be

Hoffmann-La Roche AG, Dr. med. H.-U. Jelitto

Tel. +49 7624 14-2400, Fax -3366
Schering Deutschland GmbH. Der Einsatz der Radionuklidtherapie mit Samarium-153-EDTMP (Quadramet ${ }^{\circledR}$ ) kann zu einer effektiven Schmerztherapie bei osteoblastischen Knochenmetastasen beitragen. Es handelt sich um eine kostengünstige Therapieoption, die zudem noch den Vorteil einer langen Wirkdauer bietet. Weitere Informationen bei

Schering Deutschland $\mathrm{GmbH}$, Gabriele Kainzbauer Tel. +49 30 34989-257

E-mail gabriele.kainzbauer@schering.de
Astra Zeneca GmbH. Auf dem ASCO (American Society of Clinical Oncology)-Meeting wurden erste Daten zur Behandlung des metastasierten Mammakarzinoms prämenopausaler Frauen mit der Kombination Fulvestrant + Goserelin vorgestellt. In der Kombination mit Goserelin, das prämenopausale Frauen in eine artifizielle Postmenopause versetzt, zeigt Fulvestrant einen klinischen Nutzen, der mit den Ergebnissen bei postmenopausalen Frauen vergleichbar ist.

Weitere Informationen bei

AstraZeneca GmbH, Martin Ehmer

Tel. +49 4103 708-3927, Fax -7 3927

E-mail martin.ehmer@astrazeneca.com

\begin{tabular}{ll}
\hline KARGER & @ 2005 S. Karger GmbH, Freiburg \\
& \\
$\begin{array}{l}\text { Fax + 49 761 4 52 07 14 } \\
\begin{array}{l}\text { E-mail Information@Karger.de } \\
\text { www.karger.com }\end{array}\end{array}$ & $\begin{array}{l}\text { Accessible online at: } \\
\text { www.karger.com/onk }\end{array}$ \\
&
\end{tabular}




\section{GlaxoSmithKline}

\section{Lapatinib - Tyrosinkinase-Inhibitor mit hoher First-line-Aktivität beim Mammakarzinom}

Lapatinib ist ein neuer oraler Tyrosinkinase-Hemmer mit vielversprechenden Therapieergebnissen, erläuterte Prof. George Sledge, Indianapolis/USA anlässlich der diesjährigen Jahrestagung der Amerikanischen Gesellschaft für Klinische Onkologie (ASCO) in Orlando. Als First-line-Monotherapie erreicht Lapatinib bei Patientinnen mit fortgeschrittenem und metastasiertem, Erb1- und ErbB2-überexprimierendem Mammakarzinom eine Ansprechrate von $35 \%$. Es traten keine schweren Nebenwirkungen auf. Die kardiale Verträglichkeit war gut. Laut Sledge könnte Lapatinib zukünftig eine wichtige Therapieoption für die First-line-Behandlung von Patientinnen mit ErbB1/2-überexprimierendem Mammakarzinom sein. Gegenüber dem bereits zugelassenen monoklonalen Antikörper Trastuzumab hat Lapatinib den Vorteil, nicht nur die Aktivität des ErbB2-Rezeptors (HER2/neu), sondern auch jene des ErbB1-Rezeptors (EGFR) zu blockieren. Beide Rezeptoren spielen eine wichtige Rolle beim Tumorwachstum, der Tumorinvasion und der Metastasierung. Lapatinib greift auf zwei Wegen in die Signaltransduktionskaskade der Tumorzellproliferation ein. Einen weiteren Vorteil von Lapatinib sieht Sledge in der oralen Gabe, die für die Patienten wesent- lich angenehmer ist als eine Infusion und die Behandlung erleichtert.

\section{Lapatinib - hohe First-line-Aktivität}

Die Ergebnisse der randomisierten PhaseII-Studien bestätigen sowohl die gute Verträglichkeit als auch eine hohe Wirksamkeit von Lapatinib. 130 Patientinnen mit fortgeschrittenem Mammakarzinom und zum Teil ausgeprägter - auch viszeraler Metastasierung werden randomisiert und mit zwei verschiedenen Dosierungen $1.500 \mathrm{mg}$ pro Tag oder $500 \mathrm{mg} /$ BID Lapatinib - jeweils über mindestens 12 Wochen behandelt. 33\% der Patientinnen waren adjuvant und/oder neoadjuvant vorbehandelt. Von den in einer Zwischenanalyse ausgewerteten 40 Patientinnen erreichten $14(35 \%)$ eine objektive Tumorremission (Tumorregression $>30 \%$ ), bei weiteren 14 Patientinnen (35\%) stabilisierte sich die Erkrankung vorübergehend, so dass insgesamt $70 \%$ der Patientinnen von der Behandlung mit Lapatinib profitierten. Ein unabhängiges Expertengremium bestätigte die Ergebnisse. Bei insgesamt sehr guter Verträglichkeit - schwere Grad 3-4Nebenwirkungen traten nicht auf - ergaben sich leichte Vorteile für die niedrigere Dosis. Am häufigsten klagten die Patientinnen über Hautveränderungen - Juckreiz, trockene Haut, Ausschlag, Akne sowie Diarrhö, die jeweils mild bis moderat ausgeprägt waren.

Lapatinib/Trastuzumab - Synergien nutzen Präklinische Untersuchungen weisen auf synergistische Effekte zwischen Lapatinib und Trastuzumab hin, die sich jetzt in einer Phase-I-Dosiseskalationsstudie klinisch bestätigten. Trotz zum Teil intensiver Vorbehandlung, unter anderem mit einer Tras- tuzumab-haltigen Therapie, erreichten $22 \%$ (6/27) der Patientinnen unter Lapatinib/Trastuzumab eine objektive Remission, bei vier weiteren Patientinnen stabilisierte sich die Erkrankung für mindestens $6 \mathrm{Mo}-$ nate. Auf keinem Dosislevel kam es zu einem symptomatischen Abfall der LVEF. Als Dosierung für weitere Phase-II/IIIStudien empfehlen die Autoren 1.000 $\mathrm{mg} /$ Tag Lapatinib plus Trastuzumab in der Standarddosierung (Loading-Dosis 4 $\mathrm{mg} / \mathrm{kg} \rightarrow 2 \mathrm{mg} / \mathrm{kg} /$ Woche).

Prädiktive Marker für ein Therapieansprechen identifizieren

Die Studiengruppe um Blackwell et al., konnte zeigen, dass Trastuzumab-refraktäre Mammakarzinom-Patientinnen mit Östrogen- und Progesteron-positivem und ErbB1überexprimierendem Tumor bevorzugt auf Lapatinib ansprechen. Dies ist ein erster Schritt, vor Therapiebeginn Biomarker zu identifizieren, die als Prädiktoren für ein Therapieansprechen dienen. Davon unabhängig bestätigte sich die gute Wirksamkeit von Lapatinib bei Patientinnen mit metastasiertem, ErbB1/2-überexprimierendem Mammakarzinom. Trotz zum Teil weit fortgeschrittener Erkrankung und intensiver Vorbehandlung - auch mit Trastuzumab waren nach 16 Wochen noch 7 von derzeit 19 auswertbaren Patientinnen progressionsfrei.

Weitere Informationen erhalten Sie auch unter www.brustkrebs-studien.com.

GlaxoSmithKline GmbH \& Co. KG

Unternehmenskommunikation

Sonja Luz

Theresienhöhe 11,

80339 München

Tel. +49 89360 44-8256, Fax -8066

E-mail Sonja.Luz@gsk.com

www.glaxosmithkline.de

\section{PharmaTicker+++ PharmaTicker+++ PharmaTicker+++ PharmaTicker+++}

Novartis Pharma GmbH. Novartis hat in der EU und den USA die Zulassung für Exjade ${ }^{\circledR}$ (Deferasirox) beantragt. Exjade ist der erste und einzige Eisenchelatbildner, der zur Behandlung von chronischer Eisenüberladung infolge von Bluttransfusionen einmal täglich oral zu verabreichen ist. Zulassungsanträge für weitere Länder folgen in Kürze.

Weitere Informationen bei

Novartis Pharma GmbH, Dr. Michaela Paudler-Debus

Tel. +49 911 273-12462, Fax -12971

E-mail michaela.paudler-debus@novartis.com
Grünenthal GmbH. Durch die Neueinführung (1. Juni 2005) des Buprenorphinhaltigen Matrixpflasters Transtec ${ }^{\circledR}$ PRO wird die Anwenderfreundlichkeit nochmals gesteigert, denn aufgrund seiner bis zu 96 Stunden anhaltenden Wirkung muss das Pflaster nur noch an zwei festen Tagen pro Woche gewechselt werden. Weitere Informationen bei Grünenthal $\mathrm{GmbH}$, Peter Engisch Fax +49 241 569-3151 E-mail Peter.Engisch@gruenenthal.de
Celgene Corporation. Auf dem 10. Kongress der European Hematology Association (EHA) in Stockholm wurden klinische Daten einer PhaseII-Studie zur Behandlung der chronischen lymphatischen Leukämie (CLL) mit Revlimid (Lenalidomid) vorgestellt, die dafür sprechen, dass Revlimid ein potenzieller neuer Ansatz zur Behandlung von Patienten mit rezidivierender oder refraktärer CLL ist.

Weitere Informationen bei

Weber Shandwick, Dr. Torsten Rothärmel

Tel. +49 69913 043-551, Fax -559

E-mail trothaermel@webershandwick.com

\begin{tabular}{|c|c|}
\hline KARGER & (C) 2005 S. Karger GmbH, Freiburg \\
\hline $\begin{array}{l}\text { Fax + } 497614520714 \\
\text { E-mail Information@Karger.de } \\
\text { www.karger.com }\end{array}$ & $\begin{array}{l}\text { Accessible online at: } \\
\text { www.karger.com/onk }\end{array}$ \\
\hline
\end{tabular}




\section{PFASLODEX}

\section{Faslodex ${ }^{\circledR}$ (Fulvestrant): Klinischer Benefit in der 2nd-line-Therapie höher als bei späteren Therapie- sequenzen}

Das klinische Ansprechen von Faslodex ${ }^{\circledR}$ ein reiner Östrogenrezeptor-Antagonist zur Behandlung des hormonsensitiven Mammakarzinoms - ist umso höher, je früher die Substanz in der endokrinen Therapiesequenz beim fortgeschrittenem Brustkrebs eingesetzt wird. Dabei ist das klinische Ansprechen als Summe aus kompletter und partieller Remission (CR + PR) und der Krankheitsstabilisierung (SD) $>6$ Monate definiert. Mehrere Studien im Vergleich zu Aromatasehemmern und Tamoxifen lieferten bereits sehr gute Effektivitätsdaten für die Therapie mit Faslodex ${ }^{\circledR}$. Das klinische Ansprechen war dabei umso höher, je früher Faslodex ${ }^{\circledR}$ zum Einsatz kam.

Neue Daten vom ASCO von Mauriac et al. bestätigen den frühen Einsatz von Faslo$\operatorname{dex}^{\circledR}$ beim chronischen Mammakarzinom. Sie behandelten 41 Patientinnen im Rahmen eines Studienprogramms, die nach vorangegangener Progression unter endokriner Therapie oder unter Chemotherapie Faslodex ${ }^{\circledR}$ erhielten.

In dieser Studie zeigten $83 \%$ der Patientinnen ein klinisches Ansprechen, die Faslo$\operatorname{dex}^{\circledR}$ als 2nd-line-Therapie erhielten. Bei Patientinnen, die Faslodex ${ }^{\circledR}$ in einem späteren Therapieschritt erhielten, lag die Erfolgsrate bei $47 \%$. Sehr gute Therapieer- folge wurde auch bei Patientinnen mit viszeralen Metastasen erzielt, $58 \%$ der Patientinnen zeigten ein klinisches Ansprechen.

Diese Resultate aus dem klinischen Alltag bestätigen die sehr guten Daten, die Faslodex ${ }^{\circledR}$ bereits in zwei großen Phase-III-Studien $(\mathrm{n}=851)$ gezeigt hat. Nach Tamoxifenbehandlung wurde Faslodex ${ }^{\circledR}$ (Fulvestrant, einmal monatlich $250 \mathrm{mg}$ i.m.) eingesetzt und war hier mindestens ebenso wirksam wie Arimidex ${ }^{\circledR}$ (Anastrozol, einmal täglich $1 \mathrm{mg}$ ), ein in dieser Indikation bereits seit Jahren etablierter nichtsteroidaler Aromatase-Inhibitor der 3. Generation. Patientinnen unter Faslodex ${ }^{\circledR}$ waren 5,5 Monate progressionsfrei gegenüber 4,1 Monaten unter Arimidex ${ }^{\circledR}$. Dieser Vorteil von Faslodex ${ }^{\circledR}$ bestätigte sich auch im objektiven Ansprechen, dem klinischen Benefit und insbesondere der Ansprechdau-

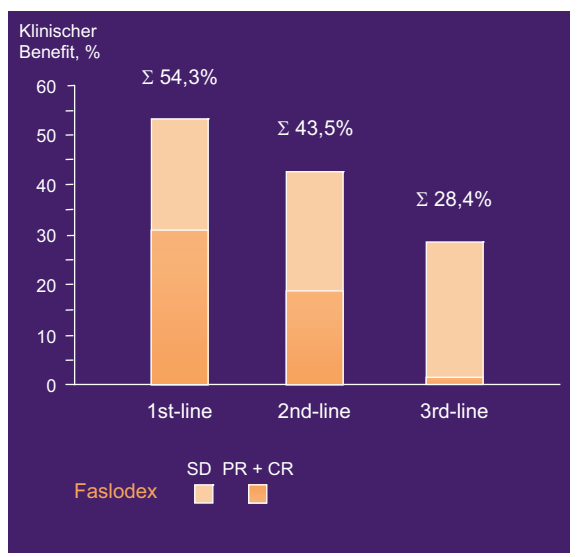

Abb. 1: Faslodex ${ }^{\circledR}$ : Je früher der Einsatz, desto höher der klinische Nutzen. (Nach Howell et al., J Clin Oncol 2004; Robertson et al., Cancer 2003; Perey et. al., San Antonio Breast Cancer Symposium 2004.) er (16,7 vs. 13,7 Monate). Auch in dieser Studie profitierten Patientinnen mit viszeralen Metastasen von der Fulvestrant-Therapie. Sie zeigten unter Faslodex ${ }^{\circledR}$ eine längere Ansprechdauer (median 17,5 vs. 11,7 Monate) und der Anteil dieser Patientinnen mit einem klinischen Benefit lag mit 49,3 gegenüber $41,9 \%$ unter Arimidex ${ }^{\circledR}$. Therapie deutlich höher.

Ingle et al. belegten im Rahmen einer weiteren Studie $(\mathrm{n}=77)$ den frühen Einsatz von Faslodex ${ }^{\circledR}$. Sie setzten Faslodex ${ }^{\circledR}$ in der 2. bzw. 3. endokrinen Therapielinie nach einem Aromatasehemmer ein und konnten dabei einen klinischen Benefit bei $52 \%$ bzw. bei $25 \%$ der Patientinnen nachweisen. Auch diese Ergebnisse sprechen für den frühen Einsatz von Faslodex und belegen gleichzeitig die exzellente Wirksamkeit von Faslodex ${ }^{\circledR}$ nach dem Einsatz eines Aromatasehemmers.

Faslodex ${ }^{\circledR}$ ist seit einem Jahr in Deutschland für die palliative Therapie postmenopausaler Frauen zugelassen. Mehrere nationale und internationale Fachgesellschaften empfehlen Faslodex ${ }^{\circledR}$ als zusätzlichen endokrinen Therapiestandard für das chronische Mammakarzinom. Die Ärzte haben die neue Behandlungsoption bereits in ihre Routinetherapie integriert und setzen Faslodex ${ }^{\circledR}$ dabei verstärkt als 2nd-line-Therapie ein.

Weitere Informationen zu Faslodex ${ }^{\circledR}$ auf www.hcp.faslodex.de

AstraZeneca GmbH

Martin Ehmer

Tinsdaler Weg 183

22880 Wedel

Tel. +49 4103 708-3927, Fax -73927

E-mail martin.ehmer@astrazeneca.com

\section{PharmaTicker+++ PharmaTicker+++ PharmaTicker+++ PharmaTicker+++}

biolitec AG. An mehreren europäischen und asiatischen Referenzzentren konnte die Wirkung des Krebsmedikamentes Foscan $\circledast$ (Temoporfin) bei der Behandlung von Zervixkarzinomen, -dysplasien und Vulvakarzinomen bestätigt werden. Foscan ${ }^{\circledR}$ ist in Europa seit Oktober 2001 für die palliative Behandlung von fortgeschrittenen Tumoren im Hals- und Kopfbereich zugelassen.

Weitere Informationen bei

biolitec AG, Dr. Marlies Zedlacher

Tel. +49 228 97967-70, Fax -99

E-mail: ir@biolitec.de sanofi-aventis. Im N Engl J Med wurde die Phase-III-Studie BCIRG 001/TAX 316 veröffentlicht: Bei Frauen mit operablem nodalpositivem Brustkrebs reduzierte die adjuvante Behandlung mit Taxotere ${ }^{\circledR}$ (Docetaxel) die Rückfallrate signifikant um $28 \%(p=0,001)$; die Mortalitätsrate um $30 \%(p=0,008)$, unabhängig vom Hormonrezeptor-, menopausalen und HER-2/neuStatus (Follow-up: 55 Monate).

Aventis Pharma Deutschland GmbH -

Ein Unternehmen der sanofi-aventis Gruppe Cristina lannazzo

Tel. +49 69 305-24417, Fax -18802

E-mail cristina.iannazzo@sanofi-aventis.com
RINECKER PROTON THERAPY CENTER. In wenigen Wochen wird Europas erstes vollklinisches Protonentherapie Zentrum in München eröffnen. Im Vergleich zur Röntgentherapie bietet die Bestrahlung mit Protonen bessere Heilungschancen bei Tumoren. Die herausragenden Vorteile der Protonen sind ihre Zielgenauigkeit und die geringen Nebenwirkungen.

Weitere Informationen bei

Pleon Healtcare $\mathrm{GmbH}$, Dr. Stefanie Wesche

Tel. +49 89590 42-200, Fax -237

E-mail Stefanie.Wesche@pleon.com

\section{KARGER ๑ $2005 \mathrm{~s}$. Karger GmbH, Freiburg

